\title{
A Rare Case of a Transphyseal Distal Humerus Fracture in a Pediatric Patient
}

\section{Victoria I Clark, Rolanda A Willacy*, Arthur R McDowell and Robert H Wilson}

Department of Orthopaediv Surgery and Rehabilitation, Howard University Hospital, USA

*Corresponding author: Rolanda A Willacy, Department of Orthopaediv Surgery and Rehabilitation, Howard University Hospital, Washington, USA, Tel: 2028656100; Email: rkingst00@gmail.com

\section{Case Report}

Volume 5 Issue 1

Received Date: March 12, 2021

Published Date: April 07, 2021

DOI: $10.23880 /$ jobd-16000207

\section{Abstract}

Birth injuries are uncommon and occur in less than $1 \%$ of the population. Transphyseal fractures of the distal humerus typically occur in children younger than three years old and may result from birth trauma in cases where excessive traction or obstetrical maneuvers are required. Early and accurate diagnosis is necessary for successful management. Differential diagnosis includes non-accidental trauma, brachial plexus injury, elbow dislocation, septic arthritis and osteomyelitis. This case report describes a rare case of a transphyseal distal humerus fracture in a pediatric patient.

Keywords: Transphyseal distal humerus, pediatric, brachial plexus injury, cartilage, traumatic injury, obstetric maneuvers

\section{Introduction}

Birth injuries occur in less than $1 \%$ of the population and are overall uncommon [1]. Transphyseal fractures of the distal humerus typically occur in children younger than 3 years old and may be secondary to birth trauma in cases where excessive traction is applied, the birth is traumatic, or the delivery requires obstetrical maneuvers $[2,3]$. Other causes of transphyseal fractures of the distal humerus include nonaccidental trauma and fall from a low level height [4-7]. Successful management is dependent on an early and accurate diagnosis of the injury, through clinical and radiologic evaluation. While transphyseal distal humerus fractures are rare, the true incidence may be underrepresented due to missed diagnoses. Here, we report a case that was originally considered a brachial plexus injury but later found to be a neonatal transphyseal distal humeral fracture.

\section{Case Report}

A newborn girl, born at 39 weeks and 7 pounds 4 ounces via normal spontaneous vaginal delivery after an uncomplicated pregnancy, was noted in the newborn nursery to have less movement in the left upper extremity in comparison to the right upper extremity. Nine days later, the patient was evaluated by her pediatrician and it was again noted that the patient was not moving the left upper extremity. Although the delivery was not noted to be particularly traumatic, there was concern for a brachial plexus injury and the child was referred to neurology one week later for evaluation. No radiographs were obtained.

On physical examination, she was noted to move both upper extremities spontaneously, although she did move the right upper extremity more frequently. She was observed to actively flex and extend the elbow, although she lacked 10 degrees of full extension. Active flexion, pronation, and supination were full and her grasp reflex was intact. There was full passive range of motion at the shoulder, wrist and hand. She was non-tender to palpation in the antecubital fossa; however a large mass was noted.

The patient was referred to neurology and was scheduled for evaluation two weeks later. The initial impression was that this was a brachial plexus injury. Since the injury 
was present at birth, non-accidental was highly unlikely. However, given the antecubital mass, plain radiographs of the left elbow were obtained and demonstrated a left elbow physeal separation with significant callus formation (Figure 1). The patient returned for a follow-up visit two weeks later, and radiographs confirmed posterior transphyseal distal humeral separation with bony callus formation (Figure 2). She was referred to orthopaedics for definitive management, was treated with cast immobilization, and ultimately went on to union without complication. During the two-month clinical visit, that patient was, the physical examination revealed that patient had near full pronation and supination and approximately 95 degrees of flexion. Orthogonal images were then obtained which demonstrated near normal anatomic alignment (Figure 3). The plan was made by the orthopaedic surgeon to continue watchful waiting and a two-month follow-up was scheduled. During the four month visit, the patient had full range of motion and function of the left arm. Evaluation of the radiographs revealed a healed and remodeled left transphyseal distal humerus fracture, with posterior bowing noted (Figure 4). A follow-up was scheduled 1-year later to screen for premature growth arrest around the elbow examines arm function.

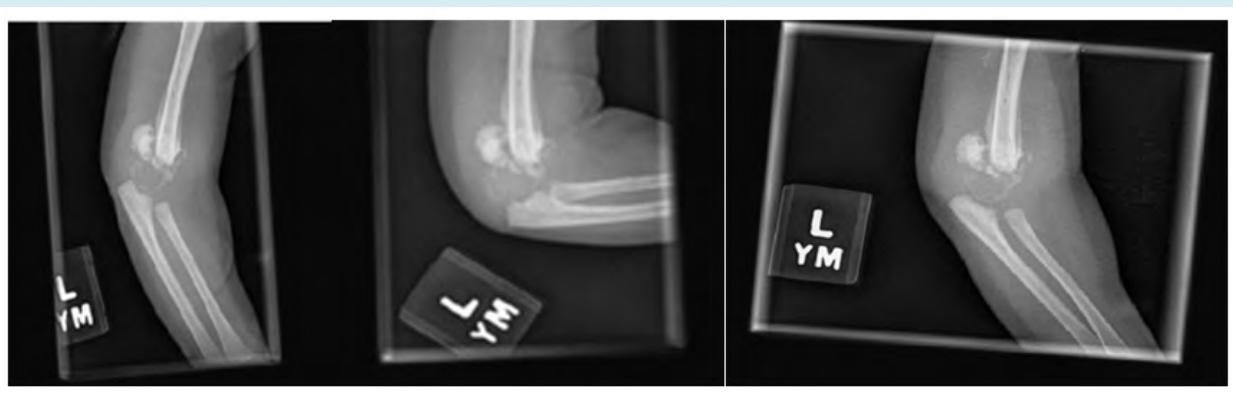

Figure 1: Anteriorposterior (AP), lateral and oblique radiographs taken two weeks after birth. Radiographs reveal a left elbow physeal separation with significant callus formation.

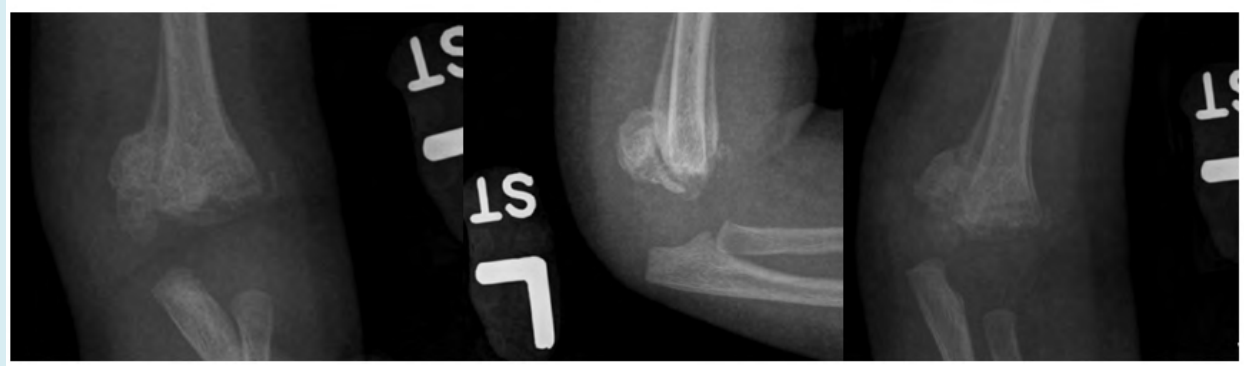

Figure 2: Anteriorposterior (AP), lateral and oblique radiographs confirm posterior transphyseal distal humeral separation with bony callus formation at 2 months.

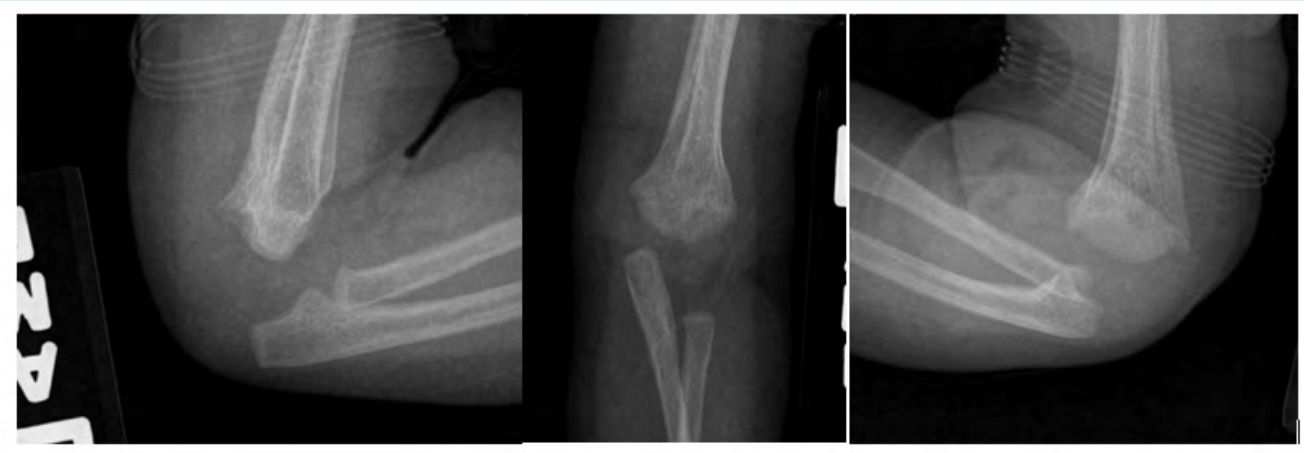

Figure 3: Orthogonal images demonstrate near normal anatomic alignment at the 2 month visit. 


\section{Journal of Orthopedics \& Bone Disorders}

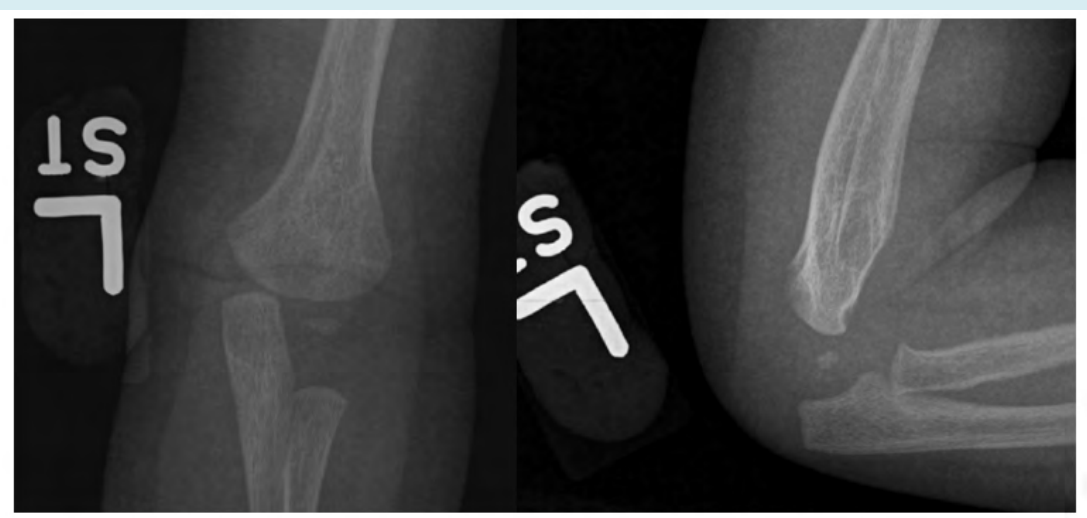

Figure 4: Anteriorposterior (AP) and lateral views reveal healed and remodeled left transphyseal distal humerus fracture, with posterior bowing.

\section{Discussion}

In this case, a brachial plexus injury was initially considered. Radiographs were obtained due to the large mass presentin theantecubitalregion and the patientwasdiagnosed with a transphyseal distal humerus fracture, however the diagnosis was delayed over two weeks. Unfortunately, this is not uncommon with this fracture pattern. Transphyseal distal humerus fractures are an uncommon injury in neonates but must remain a consideration. Early and accurate diagnosis is important, as the differential diagnosis includes fracture of the lateral humeral condyle, brachial plexus injuries and elbow dislocation.

Clinical findings include swelling around the elbow, abnormal motion, limited use of the involved upper extremity and crepitus with manipulation. Radiographic evaluation is essential to diagnosis, although there are limitations to the use of plain radiographs as the distal humerus is mainly comprised of cartilage in this age group. As a result, these injuries are often misdiagnosed and cannot be distinguished from elbow dislocations $[8,9]$.

Separation of the distal humeral epiphysis as a result of trauma during birth is both rare and difficult to correctly diagnose. Most patients presenting with this birth injury have a complicated birth history, however our case illustrates that this is not always the case. Generally, emergency cesarean deliveries, delivery of large or breech neonates, or shoulder dystocia requiring obstetric maneuvers and/or significant traction can result in a traumatic separation of the distal humeral epiphysis. The mechanism of injury is related to hyperextension of the elbow or a backward force on the forearm while the elbow is in a flexed position [10].

In considering the diagnosis of transphyseal fractures of the humerus, a differential diagnosis of osteomyelitis and septic arthritis should also be considered. Other possible causes for this presentation may include osteogenesis imperfect and other metabolic bone diseases. A high level of clinical suspicion must be present when considering a neonate with limited range of motion, swelling, decreased use of an upper extremity, and crepitus with range of motion. If there is suspicion of a transphyseal distal humerus fracture, initial evaluation should include radiographs of the affected elbow.

Diagnosis with radiographs continues to pose challenges as the ossification center in neonates is not visible until 3-9 months after birth, but radiographs will show an abnormal relationship between the radioulnar complex and the humeral metaphysis. There is evidence to support the use of ultrasonography [11] and magnetic resonance imaging [12] as alternative imaging modalities for accurate diagnosis. Ultrasound allows for detailed imaging of the cartilaginous epiphysis and can reveal periosteal elevation that results from fracture. Magnetic resonance imaging can allow for visualization of the cartilaginous epiphysis in multiple planes, allowing for more complete assessment and diagnosis of the fracture. The major pitfall to MRI in neonates however, is the frequent need for general anesthesia in order to get a high quality study [12].

Separation of the distal humeral epiphysis as a result of trauma during birth is both rare and difficult to correctly diagnose. Our case report highlights the difficulty in diagnosis of a transphyseal distal humerus fracture and should be taken as a reminder that a high level of suspicion must exist for this type of injury. Neonates with signs and symptoms of a distal humerus transphyseal fracture should be evaluated with radiographs and, if necessary, ultrasound and possibly MRI until a clear diagnosis is established. Prompt and accurate diagnosis is essential to the proper management of these injuries and good prognostic outcomes for the patient. 


\section{Journal of Orthopedics \& Bone Disorders}

\section{References}

1. Morris S, Cassidy N, Stephens M, McCormack D, McManus F (2002) Birth-associated femoral fractures: incidence and outcome. J Pediatr Orthop 22(1): 27-30.

2. Gigante C, Kini SC, Origo K, Andrea Volpin (2017) Transphyseal separation of the distal humerus in newborns. Chin J Traumatol 20(3): 183-186.

3. Supakul N, Hicks RA, Caltoum CB, Karmazyn B (2015) Distal humeral epiphyseal separation in young children: An often- missed fracture-radiographic signs and ultrasound confirmatory diagnosis. AJR Am J Roentgenol 204(2): W192-W198.

4. Gilbert SR, Conklin MJ (2007) Presentation of distal humerus physeal separation. Pediatr Emerg Care 23(11): 816-819.

5. Shrader MW (2008) Pediatric supracondylar fractures and pediatric physeal elbow fractures. Orthop Clin North Am 39(2): 163-171.

6. Hansen M, Weltzien A, Blum J, Botterill NJ, Rommens PM (2008) Complete distal humeral epiphyseal separation indicating a battered child syndrome: A case report. Arch Orthop Trauma Surg 128(9): 967-972.

7. Abzug JM, Hot CA, Ritzman TG, Brian K Brighton (2016) Transphseal fracture of the distal humerus. J Am Acad Orthop Surg 24(2): e39-e44.

8. Raupp P, Haas D, Lovasz G (2002) Epiphyseal separation of the distal humerus. J Perinat Med 30(6): 528-530.

9. Sawant MR, Narayanan S, O’Neill K, Hudson I (2002) Distal humeral epiphysis fracture separation in neonates - diagnosis using MRI scan. Injury 33(2): 179-181.

10. Sherr-Lurie N, Bialik GM, Ganel A, Amos Schindler, Uri Givon (2011) Fractures of the humerus in the neonatal period. Isr Med Assoc J 13(6): 363-365.

11. Navallas M, Díaz-Ledo F, Ares J, Amelia SánchezBuenavida, Ma Angeles López-Vilchez, et al. (2013) Distal humeral epiphysiolysis in the newborn: utility of sonography and differential diagnosis. Clin Imaging, 37(1): 180-184.

12. Baker A, Methratta ST, Choudhary AK (2011) Transphyseal fracture of the distal humerus in a neonate. West J Emerg Med 12(2): 173. 